\title{
Novo nematodeo parasito de rã sul-americana *
}

\author{
por \\ J. F. Teixeira de Freitas e Herman Lent \\ (Com 3 estampas)
}

Em Leptodactylus ocellatus L., uma das rãs mais communs na America do Sul, Walton (1935) refere a existencia de Oswaldocruzia subauricularis (Rud., 1819) e Oswaldocruzia filiformis (Goeze, 1782) e em Leptodactylus sp.? a de Oswaldocruzia pipiens Walton, 1929.

Travassos (1937) assignala uma referencia anterior que fizera (1925) de O. subauricularis em Leptodactylus ocellatus como errada, e acredila ter havido engano na determinação de $O$. pipiens por Walton.

Provavelmente as duas outras referencias de Walton não poderão ser confirmadas; não conhecemos outro autor que tenha assignalado $O$. $i$ liformis neste hospedador e, considerando que este trichostrongylideo é peculiar a animaes que não vivem na região neotropica, achamos conveniente não acceital-o. A não ser que Walton tenha determinado material de Oswaldocruzia proveniente de rãs sul-americanas, parece-nos, lambem, que a determinação de O. subauricularis é devida a Travassos (1925) e que, graças a razões expostas acima, foi considerada inexistente.

Até o momento, com exactidão, Leptodactylus ocellatus L. só possue um Trichostrongylideo: Schulzia subventricosa (Schneider, 1866).

Autopsiando, no Laboratorio de Helminthologia, algumas rãs mortas em experiencias realisadas no Laboratorio de Physiologia do Instituto Oswaldo Cruz, o prof. H. de Souza Lopes colleccionou alguns exemplares de uma especie de Oswaldocruzia que não conseguimos identificar a nenhuma das já existentes.

É ella aqui descripta em homenagem ao colleccionador do material.

\section{Oswaldocruzia lopesi $n$. sp.}

\author{
(Ests. 1 a 3 )
}

Comprimento:- Machos 4,90 a $6,14 \mathrm{~mm}$; femeas 5,48 a $8,63 \mathrm{~mm}$. Largura:- Machos 0,108 a $0,149 \mathrm{~mm}$; femeas 0,133 a $0,158 \mathrm{~mm}$.

Corpo de coloração branca, com cuticula estriada longitudinalmente. Linhas longitudinaes presentes, levemente salientes, afastadas uma da outra de

\footnotetext{
* Recebido para publicação a 2 de Julho de 1938 e dado á publicidade em Novembro de 1938.
} 
cerca de 0,010 a $0,012 \mathrm{~mm}$. Extremidade anterior afilada, geralmente curvada ventralmente, com dilatação cephalica annelada, medindo 0,078 a 0,093 mm. de comprimento por 0,040 a $0,045 \mathrm{~mm}$. de maior largura nos machos e 0,070 a $0,087 \mathrm{~mm}$. por 0,044 a $0,052 \mathrm{~mm}$. nas femeas. Bocca simples, circumdada por papillas inconspicuas. Esophago claviforme, com 0,37 a $0,43 \mathrm{~mm}$. de comprimento por 0,038 a $0,052 \mathrm{~mm}$. de largura maxima nos machos e 0,35 a 0,48 $\mathrm{mm}$. por 0,044 a $0,070 \mathrm{~mm}$. nas femeas. Annel nervoso situado logo atraz do meio do esophago, a 0,20 a $0,23 \mathrm{~mm}$. da extremidade anterior nos machos e 0,17 a $0,28 \mathrm{~mm}$. nas femeas. Póro excretor um pouco saliente, situado ao nivel da porção posterior do esophago, a 0,31 a $0,37 \mathrm{~mm}$. da extremidade cephalica nos machos e 0,25 a $0,33 \mathrm{~mm}$. nas femeas. Azas cervicaes lateraes presentes, estriadas transversalmente, com cerca de 0,013 a $0,015 \mathrm{~mm}$. de largura, iniciando-se logo abaixo da dilatação cephalica e se extendendo até abaixo do fim do esophago, continuando-se, então, por uma linha longitudinal da cuticula. Papillas cervicaes presentes, alojadas nas azas cervicaes e situadas a 0,34 a $0,39 \mathrm{~mm}$. da extremidade anterior nos machos e 0,28 a $0,35 \mathrm{~mm}$. nas femeas.

Femeas didelphas, amphidelphas, com vulva situada na metade posterior do corpo, abrindo-se por uma fenda transversal situada no fundo de uma depressão circular do corpo de cerca de $0,03 \mathrm{~mm}$. de diametro, distante 2,07 a $2,90 \mathrm{~mm}$. da extremidade posterior. Ovejector com vagina curta e ramos divergentes, medindo approximadamente $0,52 \mathrm{~mm}$. de vestibulo a vestibulo. Utero anterior dirigido para diante, dobrando-se em U acima do fim do esophago. Utero posterior dirigido para traz, dobrando-se em U acima da região anal. Ovos de casca fina, geralmente em moruła na occasião da postura, medindo 0,080 a $0,092 \mathrm{~mm}$. de comprimento por 0,050 a $0,059 \mathrm{~mm}$. de largura. Anus com labio anterior fracamente saliente, situado a 0,12 a $0,17 \mathrm{~mm}$. da cauda. Extremidade posterior afilada, com um espinho apical de 0,008 a $0,013 \mathrm{~mm}$. de comprimento.

Machos com bolsa copuladora bem desenvolvida, trilobada, com lóbo dorsal bem individualisado. Papillas pre-bursaes não evidenciadas. Formula bursal: raios ventraes sub-iguaes, nascem por tronco commum, caminham muito proximos, quasi contiguos; raios lateraes com tronco commum; raio lateral anterior logo se isola do tronco commum aos lateraes ficando approximadamente equidistante do ventro-lateral e do lateral médio, e termina longe da margem da bolsa; raios lateraes médio e posterior sub-iguaes, com tronco commum, contiguos; raios dorsaes com tronco commum; raios dorsaes externos nascem do dorsal, são bem desenvolvidos, alojando-se nos lóbós lateraes da bolsa copuladora e terminando um pouco afastados da margem bursal; raio dorsal forte terminando, geralmente, em 6 pontas, das quaes as externas são mais desenvolvidas e as primeiras a se destacarem do tronco, a cerca de 0,025 a $0,045 \mathrm{~mm}$. da origem dos dorsaes externos. Extremidade distal do raio dorsal susceptivel de variações (est. 3, figs. 4 a 7 ). Espiculos complexos, com 0,126 a 0,139 $\mathrm{mm}$. de comprimento, apresentando nos $2 / 3$ distaes uma série de processos reunidos por membrana hyalina. Estes processos são em numero de 4: o primeiro apresenta a porção distal curvada em arco e retorcida sobre o eixo longitudinal, sendo fortemente chitinisada; o segundo processo é rectilineo e constituido por duas partes lateraes mais espessas e uma parte central menos chitinisada, termina em ponta longa e fasciculada; o terceiro processo é rectilineo, sub-cylindrico, com base commum com o processo seguinte; finalmente, 
o quarto processo, geralmente mais longo do que o terceiro, tem tronco commum com elle, apresentando a ponta, ás vezes, espessada. Variações são frequentes nos processos 1, 3 e 4: o primeiro processo apresenta commumente dois ramos filiformes, de comprimentos extremamente variaveis. Gubernaculo ausente.

Habitat: - Intestino delgado de Leptodactylus ocellatus L.

Proveniencia: - Rio de Janeiro, Brasil.

Typos e cotypos na collecção helminthologica do Instituto Oswaldo Cruz.

\section{DISCUSSÃO TAXONOMICA}

De todas as especies do genero Oswaldocruzia Travassos, 1917, O. lopesi n. sp. mais se approxima de O. mazzai Trav., 1935 e $O$. subauricularis (Rud., 1819). Desta ullima se distingue pela presença das azas cervicaes, pelas menores dimensões dos espiculos, embora o comprimento do corpo seja semelhante em ambas, pela posição do póro excretor situado muito proximo ao fim do esophago, e pelo aspecto da região vulvar. Daquella se afasta pela presença das azas cervicaes e pelo aspecto dos processos espiculares.

\section{BIBLIOGRAPHIA}

Travassos, L.

1925. Contribuições para o conhecimento da fauna helminthologica dos batrachios do Brasil. Nematodeos intestinaes. Sciencia Medica, 3 (11) : 673-687, figs. 1-10.

1937. Revisão da familia Trichostrongylidae Leiper, 1912. Monogr. Inst. Oswaldo Cruz, 1 : VII + 512 pp., 297 ests., 1260 figs.

Walton, A. C.

1935. The Nematoda as parasites of Amphibia. II. Jour. Parasit., 21 (1) : 27-50, figs. $1-4 \mathrm{~b}$. 


\section{Estampa 1}

Oswaldocruzia lopesi n. sp.

Fig. 1-Extremidade anterior do macho, vista ventral Fig. 2-Extremidade anterior da femea, vista lateral Fig. 3-Região vulvar.

Fig. 4-Aspecto do orificio vulvar, visto de frente Fig. 5-Cauda da femea. 


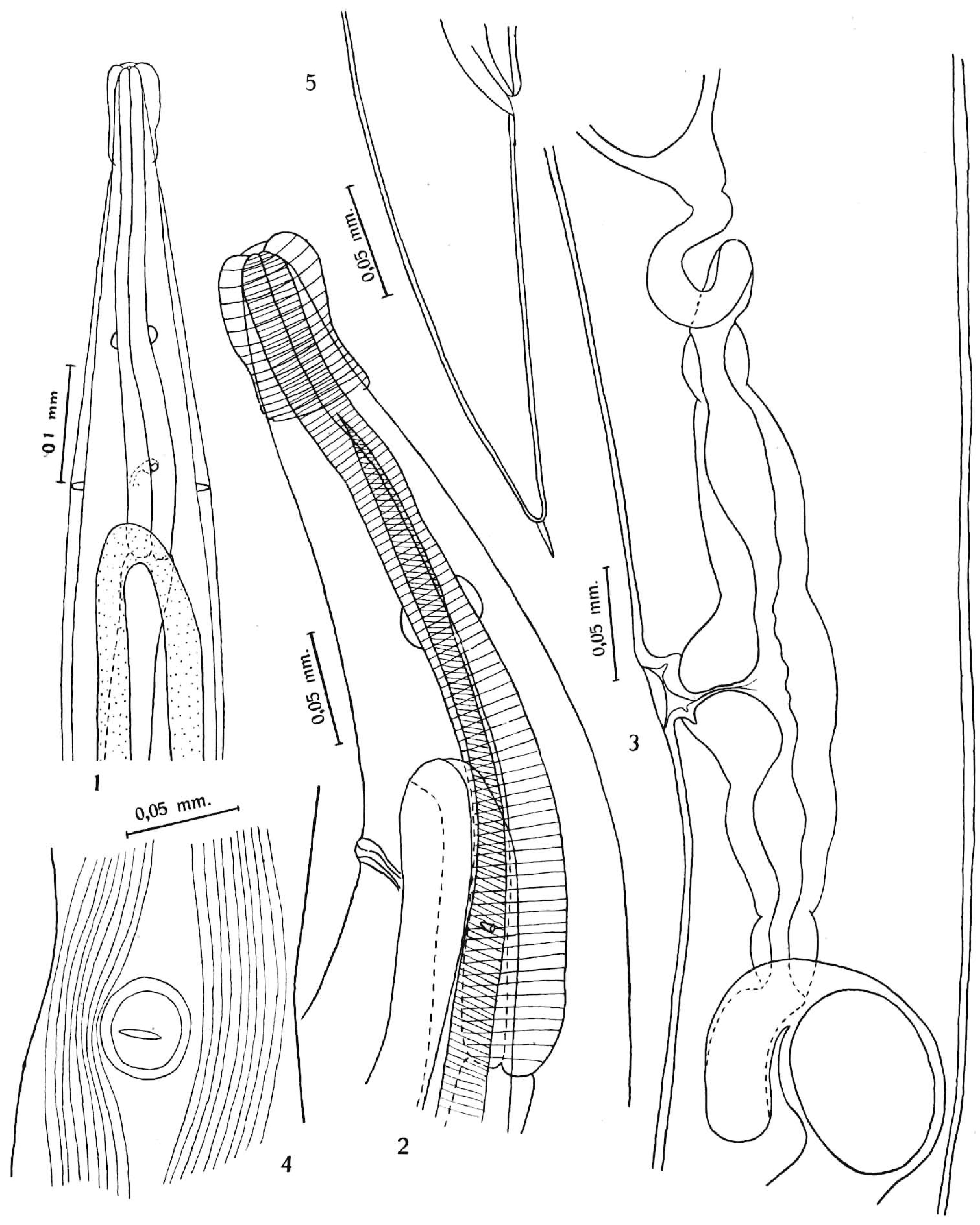

Freitas \& Lent: Oswaldocruzia lopesi n. sp. 


\section{Estampa 2}

Oswaldocruzia lopesi n. sp.

Fig. 1 -- Cauda do macho. 


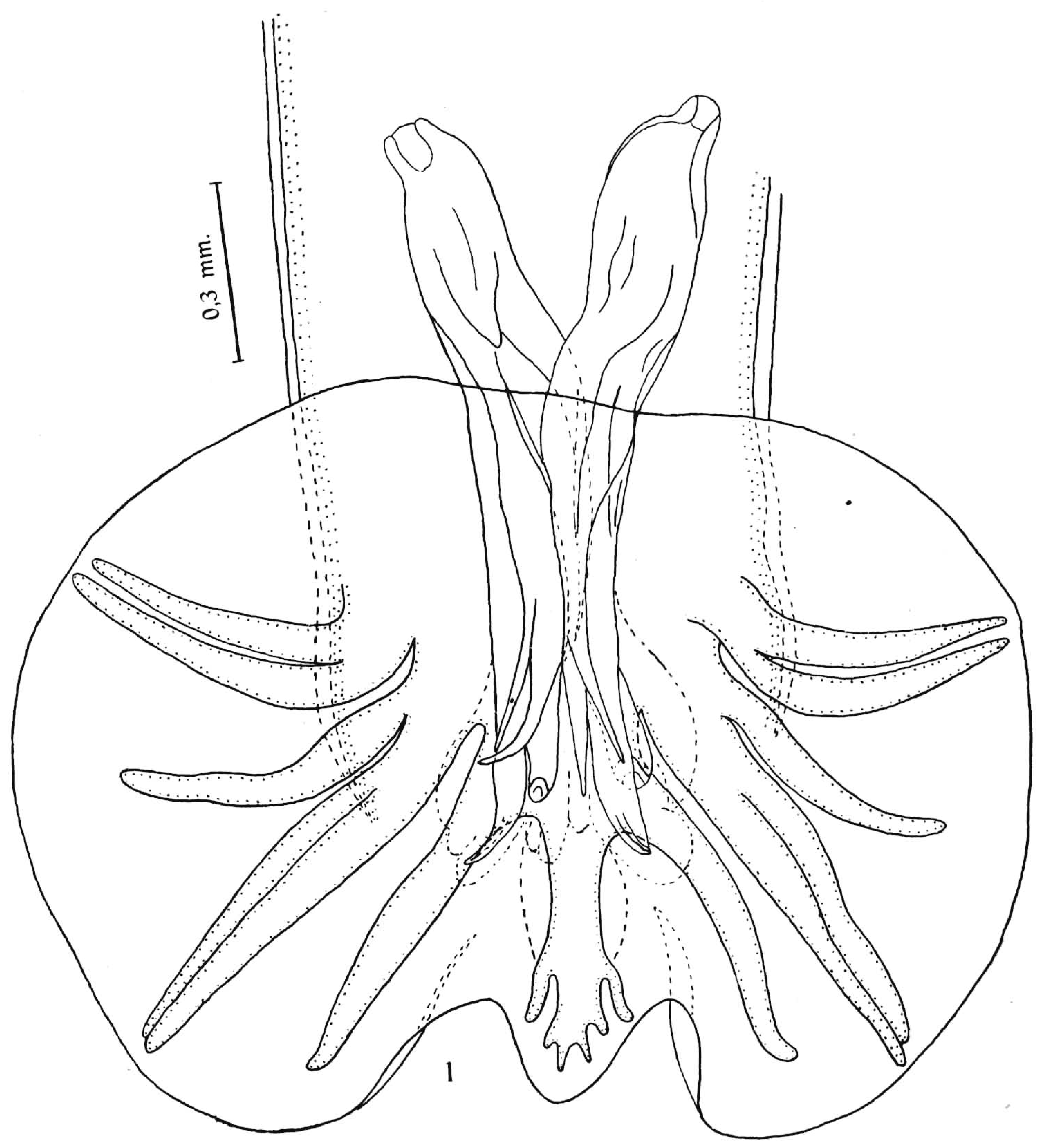

Freitas \& Lent: Oswaldocruzia lopesi n. sp. 


\section{Estampa 3}

Oswaldocruzia lopesi n. sp.

Fig. 1 - Espiculos (par).

Fig. 2-Extremidade distal dos espiculos (par)

Fig. 3 - Variação de um dos processos espiculares, observala no mesmo par.

Figs. 4 a 7 - Variações do raio dorsal da bolsa copuladora 
MEM. INST. OSWALD CRUZ

33, 4, NOV., 1938
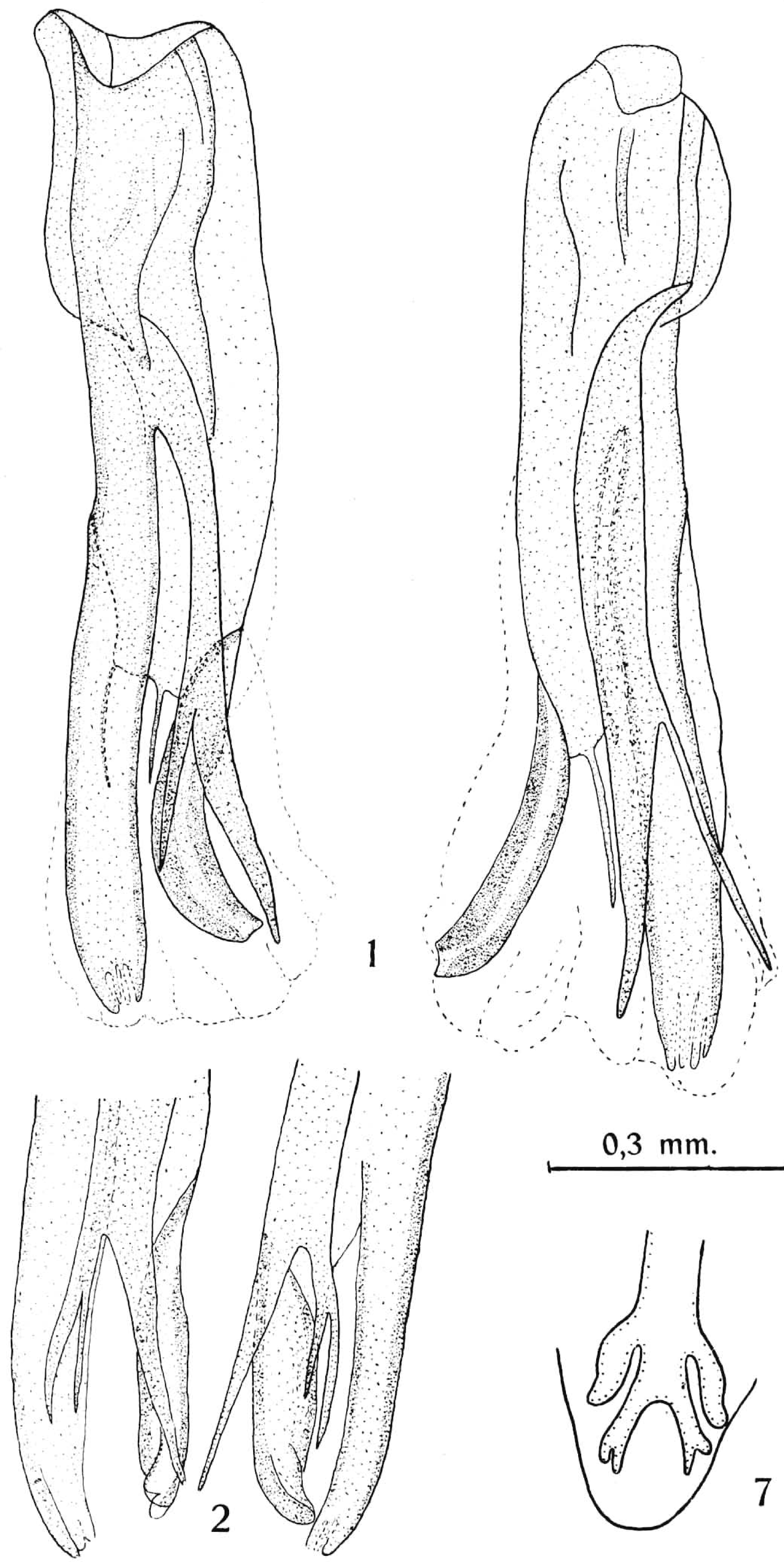

EST. 3
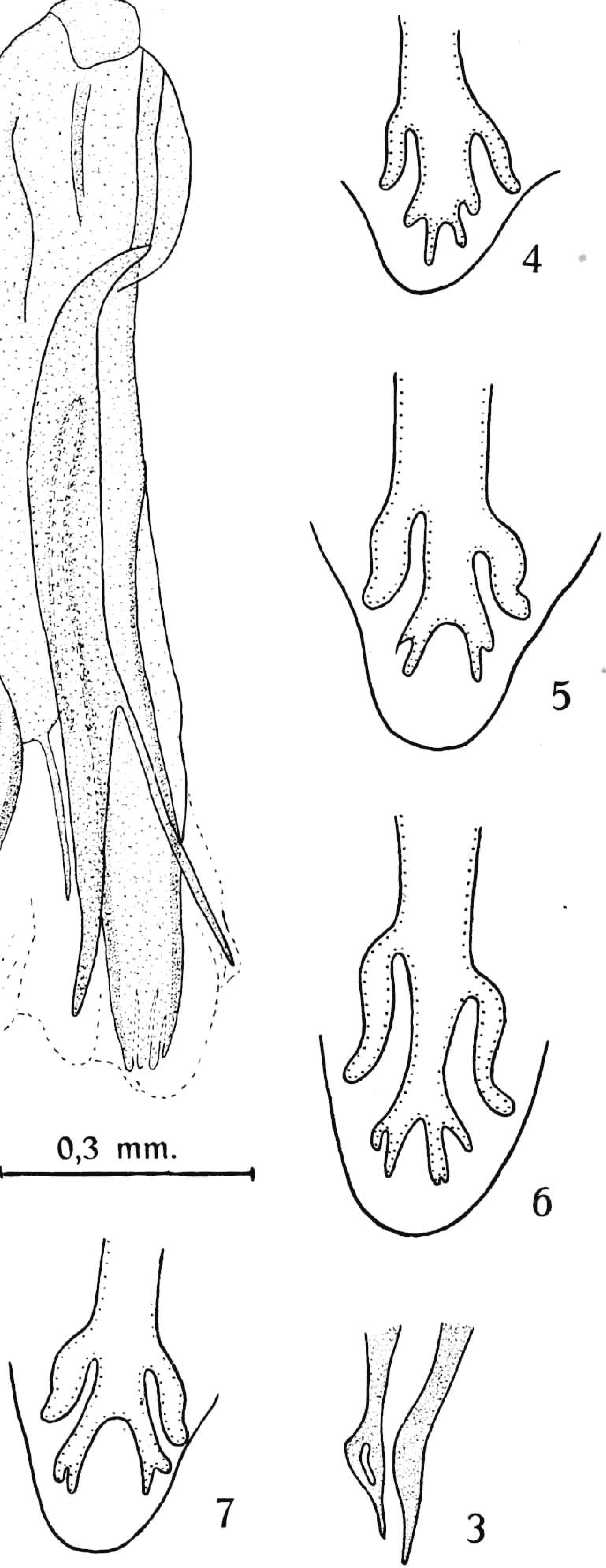

Freitas \& Lent: Oswaldocruzia lopes n. sp. 